Supplementary Information

\title{
Microfluidic device for the measurement of amino acid secretion dynamics from murine and human islets of Langerhans
}

\author{
Xue Wang, Lian Yi, and Michael G. Roper* \\ Department of Chemistry and Biochemistry, Florida State University, \\ 95 Chieftain Way, Tallahassee, FL 32306
}

\begin{abstract}
: from human islets are shown in Figure S-3.

*Address Correspondence to:

Dr. Michael G. Roper

Department of Chemistry and Biochemistry

Florida State University

95 Chieftain Way

Dittmer Building

Tallahassee, FL 32306

$\mathrm{Ph}$ 850-644-1846

Fx 850-644-8281

E-mail: roper@chem.fsu.edu
\end{abstract}

The method used to optimize reagent channel lengths for obtaining a 1:1:1 flow rate ratio of BSS:NDA:CN' is described. Electropherograms of separations using 25,35 , and $45 \mathrm{mM}$ phosphate buffers are shown in Figure S-1. Figure S-2 demonstrates the response of Leu, Trp, and Phe secretion from 2 murine islets under changing glucose concentration. The secretion profiles of 8 amino acids 


\section{Optimization of channel lengths.}

The flow rates in the three reagent channels (BSS, NDA, and $\mathrm{CN}^{-}$) were optimized to achieve a mixing ratio of $1: 1: 1$. Due to the EOF mobility difference of all 3 buffers, the electric field of the 3 channels must be modified to offset the mobility differences to obtain equal flow rates from each channel. The EOF mobilities of the 3 reagent solutions were measured on a commercial CE instrument (Beckman MDQ, Beckman Coulter, Inc., Brea CA) under the same conditions. A neutral marker, BODIPY 493/505, was utilized to calculate the EOF velocity of each buffer. The velocity ratio of BSS : NDA buffer (15 mM borate, $20 \mathrm{mM}$ EDTA with $50 \% \mathrm{ACN}$ ) : $\mathrm{CN}^{-}$buffer (15 mM borate, $20 \mathrm{mM}$ EDTA) was determined to be 1.5:1.2:1. With this ratio, the electric fields of the three reagent channels were optimized by changing the length ratio of the three channels to 0.67:0.83:1. 


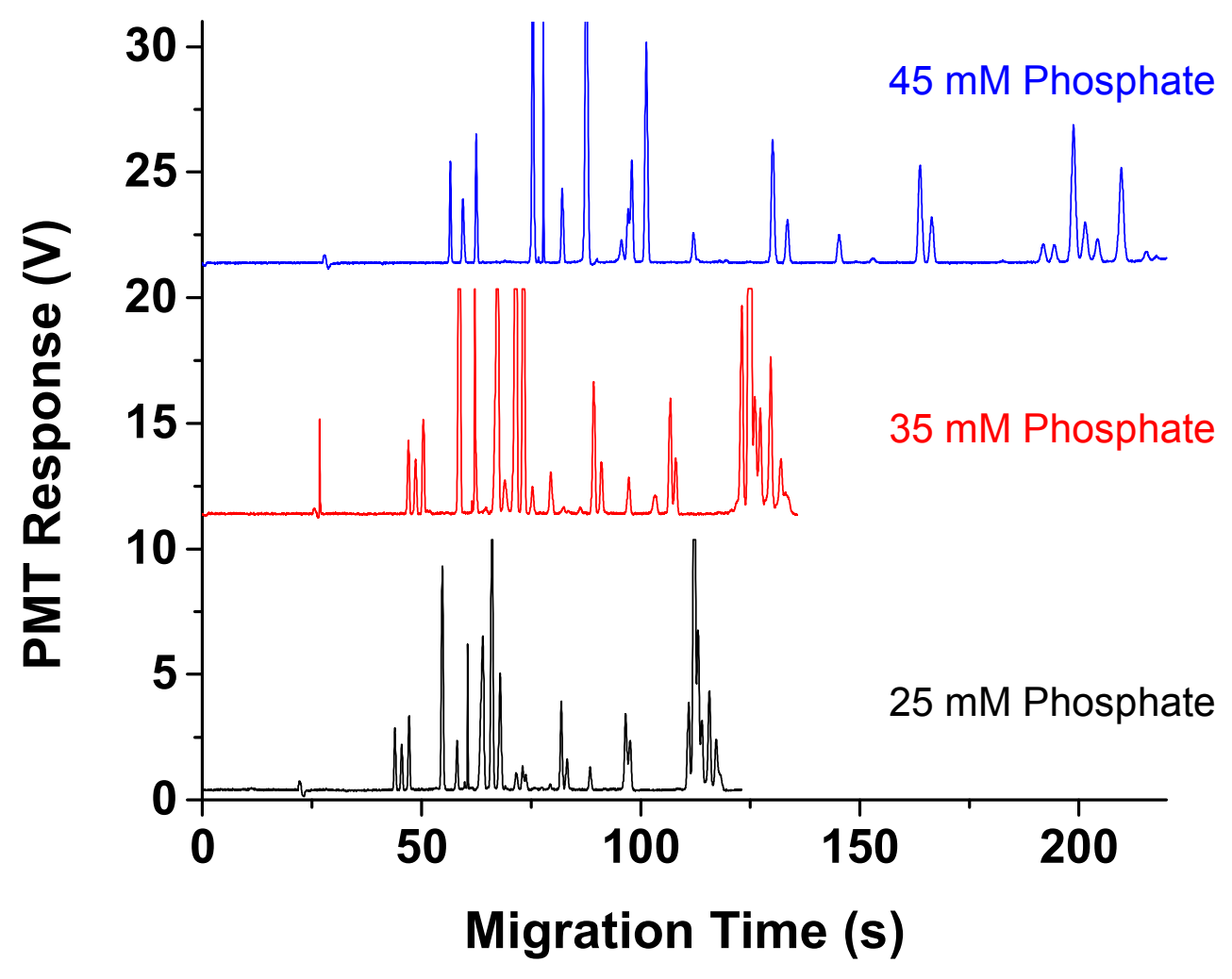

Figure S-1. Optimization of separation buffer concentration. Three phosphate concentrations were tested for resolving the most amino acids in the shortest amount of time: $25 \mathrm{mM}$ (bottom electropherogram, black), $35 \mathrm{mM}$ (middle electropherogram, red), and $45 \mathrm{mM}$ (top electropherogram, blue). All buffers were at $\mathrm{pH} 8.30$ and contained 30 $\mathrm{mM}$ SDS. The separation resolution was improved as the phosphate concentration increased from 25 to $45 \mathrm{mM}$; however, due to the increased the separation times with increased phosphate concentrations, $35 \mathrm{mM}$ was chosen as optimal. 


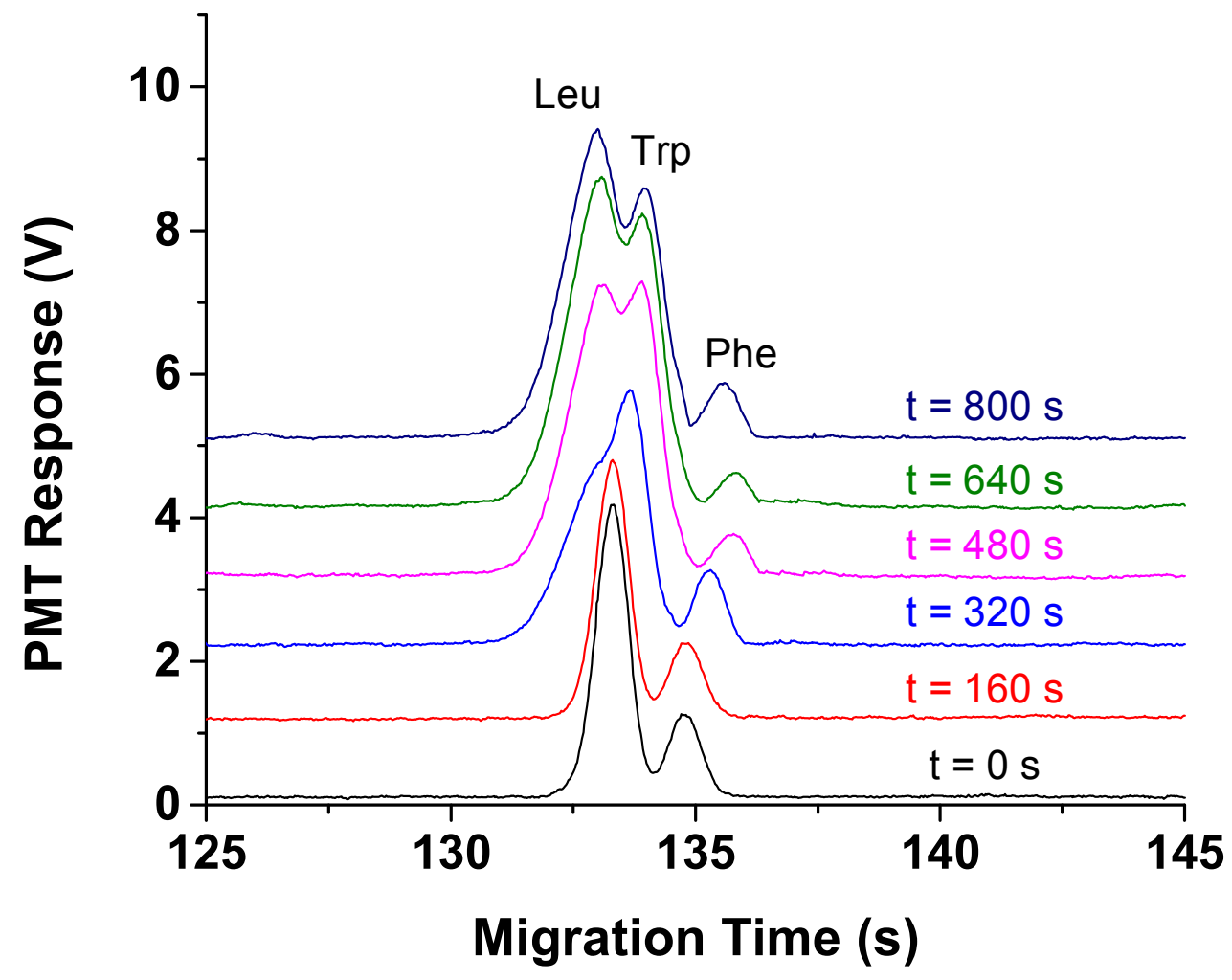

Figure S-2. Electropherograms of Leu, Trp, and Phe under changing glucose concentrations. Secretions were measured from 2 murine islets when the perfused glucose concentration was switched from 3 to $20 \mathrm{mM}$ at $\mathrm{t}=0 \mathrm{~s}$. Changes in the relative peak heights of Leu and Trp were observed as the glucose concentration was increased over time. The separation conditions were the same as those described in Figure 2. 


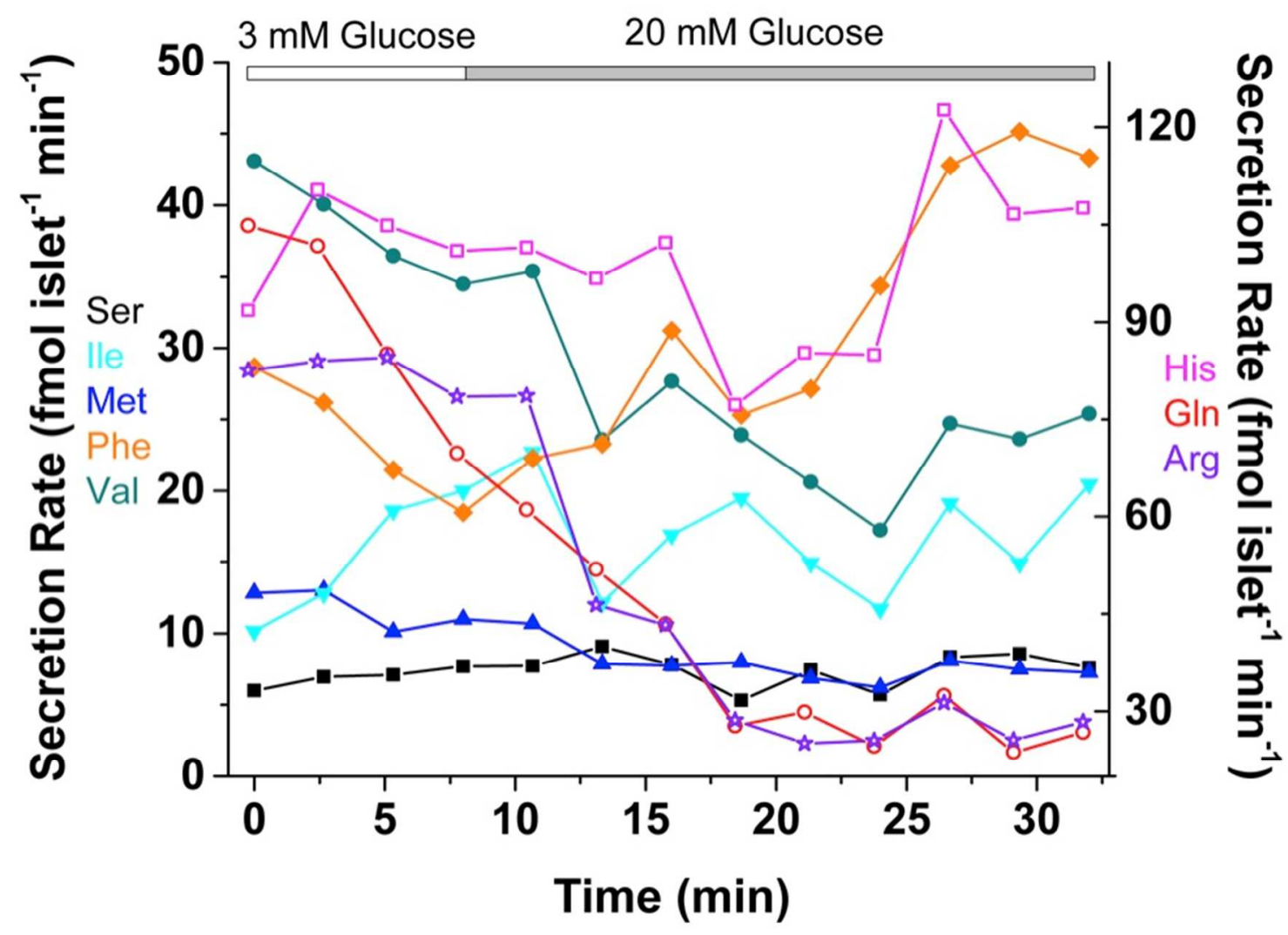

Figure S-3. Simultaneous monitoring of the secretion profiles of 8 amino acids from human islets. Secretions from 4 human islets were measured as the glucose concentration changed from 3 to $20 \mathrm{mM}$. The secretion rates of Ser ( $\boldsymbol{\square})$, lle ( $\boldsymbol{\nabla})$, Met ( $\boldsymbol{\Delta}$ ), Phe ( ), and $\operatorname{Val}(-)$ were plotted on the left y-axis. The secretion rates of His $(\square)$, Gln $(\bigcirc)$, and $\operatorname{Arg}(i)$ were plotted on the right y-axis. The perfused glucose concentration is shown on the top of the graph. The separation conditions were the same as those described in Figure 2. 